\title{
Sensing Quasi-Static Aberrations of Adaptive Optics Systems On-Line with Long-Exposure Phase Diversity
}

\author{
L. M. Mugnier ${ }^{1,2, a}$, J.-F. Sauvage ${ }^{1,2}$, T. Fusco ${ }^{1,2}$, S. Dandy ${ }^{1,2}$, and A. Cornia ${ }^{1,2,3}$ \\ 1 Office National d'Études et de Recherches Aérospatiales (ONERA), Optics Department, BP 72, \\ F-92322 Châtillon cedex, France \\ 2 Groupement d'Intérêt Scientifique PHASE (Partenariat Haute résolution Angulaire Sol Espace) be- \\ tween ONERA, Observatoire de Paris, CNRS and Université Paris Diderot \\ 3 LESIA, Observatoire de Paris, 5 place Jules Janssen, 92195 Meudon, France.
}

\begin{abstract}
We validate by simulations an extension of the phase diversity technique that uses long exposure adaptive optics corrected images for sensing quasi-static aberrations during the scientific observation, in particular for high-contrast imaging. The principle of the method is that, for a sufficiently long exposure time, the residual turbulence is averaged into a convolutive component of the image and that phase diversity estimates the sole static aberrations of interest. The advantages of such a procedure, compared to the processing of short- exposure image pairs, are that the separation between static aberrations and turbulence-induced ones is performed by the long-exposure itself and not numerically, that only one image pair must be processed, that the estimation benefits from the high SNR of long-exposure images, and that only the static aberrations of interest are to be estimated. Long-exposure phase diversity can also be used as a phasing sensor for a segmented aperture telescope. Thus, it may be particularly useful for future planet finder projects such as EPICS on the European ELT.
\end{abstract}

\section{Introduction}

Calibrating the quasi-static aberrations of a ground-based Adaptive Optics (AO) corrected imaging system is an important issue, especially for extreme AO high contrast instruments such as the proposed planet finder instruments for the ESO and Gemini 8-meter telescopes.

Measuring these aberrations off-line, i.e., during a day-time calibration on an internal reference source, has been successfully applied to existing systems such as $\mathrm{N} \quad$ [1,2] or Keck. The main limitations of such a procedure directly stem from its off-line nature: the aberrations located before the internal reference source are not sensed, and the aberrations may evolve between the day-time calibration and the night-time observation. These two limitations can be circumvented by an appropriate calibration performed on-line, i.e., during night-time observations, as described in the following.

A wave-front sensor (WFS) is able to measure the aberrations seen by the telescope on-line, but these consist of the sum of a turbulence-induced component, which is partially compensated for by the AO, and a static component. Because the turbulence, whether corrected or not, evolves quickly, the WFS measurements are generally performed with integration times that freeze the turbulence evolution, typically a few milli-seconds.

A focal-plane WFS is the only way to be sensitive to all aberrations down to the focal plane, and in particular to the so-called non-common path aberrations of an AO system, which motivates our choice for a focal-plane WFS in the following. Estimating aberrations from a single focal-plane image of a point source is a difficult problem known as phase retrieval. Gonsalves [3] showed that by using a second image with an additional known phase variation with respect to the first image such as defocus, it is possible to estimate the unknown phase even when the object is extended and unknown. This technique, referred to as phase diversity, has been significantly developed in the past twenty years, both for wave-front sensing and for imaging; see for instance Ref. [4] for a review.

The estimation of static aberrations from a series of short-exposure phase-diversity data has been performed using a series of image pairs of an astronomical object [5,6]. Lee et al. [7] have performed

\footnotetext{
a e-mail: mugnier@onera.fr
}

This is an Open Access article distributed under the terms of the Creative Commons Attribution-Noncommercial License, which permits unrestricted use, distribution, and reproduction in any noncommercial medium, provided the original work is properly cited. 
such a calibration of static aberrations with a series of images instead of image pairs, and an original diversity: no additional defocused image was used, and successive changes to the adaptive optics introduced the required diversity.

In both approaches, the static aberrations are obtained as an empirical average of the phase estimates corresponding to each short-exposure data. This is notably suboptimal for at least three reasons: (1) the images correspond to short integration times, and are consequently noisier than the corresponding long-exposure image pair, so each phase estimate suffers from this noise; (2) the computational cost is high because many short-exposure images must be processed in order to estimate the sought static aberrations; (3) the phase estimation accuracy may be penalized because the estimation must be performed on a number of phase parameters that is large enough to describe the short-exposure phase, whereas only a smaller number of these parameters may be of interest, if the sought static aberrations are of lower order than turbulence-induced ones.

In this communication, we validate a recently proposed [8] extension of the phase diversity technique that uses long-exposure AO-corrected images for sensing quasi-static aberrations. This way, (1) the separation between quasi-static aberrations and turbulence-induced ones is performed by the longexposure itself and not numerically, (2) only one image pair must be processed, (3) the estimation benefits from the high SNR of long-exposure images, and (4) only the static aberrations of interest are to be estimated.

\section{Principle of long-exposure phase diversity}

We consider a ground-based telescope observing Space through the atmosphere. The long-exposure optical transfer function (OTF) of the atmosphere+instrument system is the product of the OTF of the sole instrument $\tilde{h}_{\mathrm{s}}$, called static OTF in the following, by the atmospheric transfer function (ATF) $\tilde{h}_{\mathrm{a}}$ :

$$
\left\langle\tilde{h}_{\mathrm{o}}\right\rangle=\tilde{h}_{\mathrm{s}} \tilde{h}_{\mathrm{a}} .
$$

The static OTF is a function of the unknown static aberrations, which are coded in the phase function $\varphi$ in the aperture; let $P$ be the indicator function of the aperture, $\tilde{h}_{\mathrm{s}}$ is given by:

$$
\tilde{h}_{\mathrm{s}}(\varphi)=P e^{i \varphi} \otimes P e^{i \varphi}
$$

where $\otimes$ denotes auto-correlation. The phase function $\varphi(u, v)$ is expanded on a basis $\left\{b_{k}\right\}$, which is typically either Zernike polynomials or the pixel indicator functions in the aperture: $\varphi(u, v)=\sum_{k} \phi_{k} b_{k}(u, v)$, where the summation is, in practice, limited to the number of coefficients considered sufficient to correctly describe the static aberrations to be estimated. We shall denote by $\phi$ the vector concatenating the set of unknown aberration coefficients $\phi_{k}$.

Assuming phase perturbations with Gaussian statistics, the ATF at spatial frequency $\mathbf{f}$ is given by:

$$
\tilde{h}_{\mathrm{a}}(\mathbf{f})=e^{-\frac{1}{2} D_{\phi}(\lambda \mathbf{f})}
$$

where $\lambda$ is the imaging wavelength and $D_{\phi}$ is the phase structure function. If the turbulence is partially compensated by an AO system, Equations (1) and (3) remain valid [9], although slightly approximate because the residual phase after AO correction is not stationary.

With these equations in hand, and assuming that the image is not larger than the isoplanatic patch, we can now model the long-exposure image. The image is recorded by a detector such as a CCD camera, which integrates the flux on a grid of pixels. This can be conveniently modeled as the convolution by a detector PSF $h_{\mathrm{d}}$, assumed to be known in the sequel, followed by a sampling operation. Using Eq. (1), the global long-exposure PSF of the instrument is thus:

$$
h_{\mathrm{le}}=h_{\mathrm{d}} \star\left\langle h_{\mathrm{o}}\right\rangle=h_{\mathrm{d}} \star h_{\mathrm{s}} \star h_{\mathrm{a}},
$$

where $h_{\mathrm{s}}$ is the PSF due to static aberrations, given by the inverse Fourier transform of Eq (2), $h_{\mathrm{a}}$ is the atmospheric PSF given by the inverse Fourier transform of Eq. (3), and $\star$ denotes convolution. 
Due to the inevitable sampling and noise of the detection processes, the image $\boldsymbol{i}_{\mathrm{f}}$ recorded in the focal plane is the noisy sampled convolution of the long-exposure point-spread function (PSF) $h_{\mathrm{le}}$ with the observed object $o$. This model is generally approximated by a noisy discrete convolution with the sampled version $\boldsymbol{o}$ of the object $o$ :

$$
\boldsymbol{i}_{\mathrm{f}}=\boldsymbol{h}_{\mathrm{le}} \star \boldsymbol{o}+\boldsymbol{n}
$$

where $\boldsymbol{h}_{\mathrm{le}}$ is the sampled version of $h_{\mathrm{le}}$, and $\boldsymbol{n}$ is a corruptive noise process. If the noise is not additive and independent from the noiseless image, for instance if it is predominantly photon noise, then Eq. (5) should read $\boldsymbol{i}=\boldsymbol{h}_{\mathrm{le}} \star \boldsymbol{o} \diamond \boldsymbol{n}$, with the symbol $\diamond$ representing a pixel-by-pixel operation. For legibility we shall keep the additive notation. Combining Eq. (5) with the discrete counterpart of Eq. (4) yields the following discrete image model for the focused and defocused images respectively:

$$
\boldsymbol{i}_{\mathrm{f}}=\boldsymbol{h}_{\mathrm{d}} \star \boldsymbol{h}_{\mathrm{s}}^{(\boldsymbol{\phi})} \star\left(\boldsymbol{h}_{\mathrm{a}} \star \boldsymbol{o}\right)+\boldsymbol{n} \quad \text { and } \quad \boldsymbol{i}_{\mathrm{d}}=\boldsymbol{h}_{\mathrm{d}} \star \boldsymbol{h}_{\mathrm{s}}^{\left(\boldsymbol{\phi}+\boldsymbol{\phi}_{d}\right)} \star\left(\boldsymbol{h}_{\mathrm{a}} \star \boldsymbol{o}\right)+\boldsymbol{n}^{\prime},
$$

where $\boldsymbol{h}_{\mathrm{d}}, \boldsymbol{h}_{\mathrm{s}}$ and $\boldsymbol{h}_{\mathrm{a}}$ are the sampled versions of $h_{\mathrm{d}}, h_{\mathrm{s}}$ and $h_{\mathrm{a}}, \boldsymbol{\phi}_{d}$ is the known additional phase introduced in image $\boldsymbol{i}_{\mathrm{d}}$, and the superscripts on $\boldsymbol{h}_{\mathrm{s}}$ are reminders of the aberrations that enter the static PSF of each image.

Let $\boldsymbol{o}^{\prime}$ be the convolution of the atmospheric PSF with the observed object: $\boldsymbol{o}^{\prime}=\boldsymbol{h}_{\mathrm{a}} \star \boldsymbol{o}$. The phase-diversity data model of Eq. (6) is strictly identical to the one that would be obtained by imaging the pseudo-object $\boldsymbol{o}^{\prime}$ in the absence of turbulence and with the same static aberrations. Thus, all the phase estimation methods developed for short-exposure images, in which the OTF of the system is completely described by a phase function, can be applied here to estimate the sole static aberrations.

Additionally, it is well-known that, for a given noise level, the estimation quality of the aberrations in phase diversity depends on the spectral content of the observed scene. Thus, in the method described here, the estimation quality of the aberrations will depend both on the spectral content of the observed object and on the ATF, i.e., on the turbulence correction quality provided by the AO.

The appropriate implementation of this long-exposure phase diversity technique depends on the type of instrument, and is described in Ref. [8].

\section{Chosen phase estimation method}

Among all the possible estimation methods (see, e.g., Ref. [4] for a review) in this communication we choose, for simplicity, the conventional least-squares joint estimation of the phase $\boldsymbol{\phi}$ and the object $\boldsymbol{o}^{\prime}$, with a regularization on both quantities: $\left(\hat{\boldsymbol{o}}^{\prime}, \hat{\boldsymbol{\phi}}\right)=\arg \min J\left(\boldsymbol{o}^{\prime}, \boldsymbol{\phi}\right)$ with

$$
J\left(\boldsymbol{o}^{\prime}, \boldsymbol{\phi}\right)=\frac{1}{2 \sigma_{n}^{2}}\left\|\boldsymbol{i}_{\mathrm{f}}-\boldsymbol{h}_{\mathrm{d}} \star \boldsymbol{h}_{\mathrm{s}}^{(\boldsymbol{\phi})} \star \boldsymbol{o}^{\prime}\right\|+\frac{1}{2 \sigma_{n^{\prime}}^{2}}\left\|\boldsymbol{i}_{\mathrm{d}}-\boldsymbol{h}_{\mathrm{d}} \star \boldsymbol{h}_{\mathrm{s}}^{\left(\boldsymbol{\phi}+\phi_{d}\right)} \star \boldsymbol{o}^{\prime}\right\|+R_{o}\left(\boldsymbol{o}^{\prime}\right)+R_{\phi}(\boldsymbol{\phi}),
$$

where $\sigma_{n}^{2}$ and $\sigma_{n^{\prime}}^{2}$ are the noise variances of the two images, estimated beforehand. The object regularization is chosen as a quadratic function, so that the whole criterion $J$ is quadratic with respect to $\boldsymbol{o}^{\prime}$ and thus has an unique, closed-form solution $\hat{\boldsymbol{o}}^{\prime}(\boldsymbol{\phi})$ for a given phase $\boldsymbol{\phi}$. This allows one to replace the optimization of $J\left(\boldsymbol{o}^{\prime}, \boldsymbol{\phi}\right)$ with that of criterion $J^{\prime}(\boldsymbol{\phi}) \triangleq J\left(\hat{\boldsymbol{o}}^{\prime}(\boldsymbol{\phi}), \boldsymbol{\phi}\right)$, as commonly done in the unregularized case $[3,10]$.

Following the findings of Blanc [11], we under-regularize the object in order to best estimate the phase. This strategy is supported by the fact that it yields a phase estimation with satisfactory asymptotic properties, as shown in Ref. [12], and that these properties hold even if the noise is not Gaussian.

Concerning the phase, we choose the basis of the pixel indicator functions rather than, e.g., a truncated basis of Zernike polynomials, in order to model and reconstruct phases with a high spatial frequency content. Because of the potentially large number of phase unknowns we are lead to regularize the phase estimation. To this aim, we use a functional proposed specifically for such a phase basis [4, Sect. 8] and recalled in Ref. [8]. This regularization function has been constructed in such 
a way that it is insensitive, as the data is, to a global piston, to tip-tilt, and to any $2 \pi$ variation of the phase on any pixel. This way, no local minimum is introduced by the regularization into the minimized criterion.

\section{Validation by simulations}

We shall now validate the method by simulations. We shall essentially study the influence of the exposure time. Indeed, the main specific assumption of the method lies in Eq. (1), because the factorization of the OTF in a static OTF and an ATF is strictly valid only if the turbulence is perfectly averaged i.e., for an infinite exposure time. Note that by exposure time we mean the (finite) number of independent turbulence realizations, not the noise level. In all the simulations presented here, we have considered noiseless images. We have checked that the behavior of the phase estimation in the proposed method with respect to the noise level is not specific and is the same as conventional phase diversity with shortexposure images: the estimation error is usually proportional to the average standard deviation of the noise in the images $[13,11]$. Then we shall briefly study the influence of the AO correction quality.

\subsection{Conditions of simulation}

We consider here a point-like source, observed with an $8 \mathrm{~m}$ ground-based telescope, equipped with AO. The simulations take into account both the AO-corrected turbulence and static aberrations. The baseline adaptive optics system considered is the high-performance AO system $S$ of the $S$ instrument. We use a Fourier-based simulation method that describes the AO via the spatial power spectrum of the residual phase [14] The simulation takes the following realistic set of parameters: a $41 \times 41$ subaperture Shack-Hartmann, a $1.2 \mathrm{kHz}$ sampling frequency, a guide star of magnitude 8 , and a Paranal-like turbulence profile, with a seeing of 0.8 arcsec at $0.5 \mu \mathrm{m}$. Static aberrations are randomly generated according to a $f^{-2}$ spectrum, $f$ being the spatial frequency in the pupil, with a total wavefront error of 0.23 radian at $1.6 \mu \mathrm{m}$, i.e., $60 \mathrm{~nm}$

For some of the simulations, the number of actuators on a pupil diameter will be decreased from $40 \times 40$ to $14 \times 14$ and $7 \times 7$ in order to study the influence of the AO correction quality. The above simulation conditions lead to a phase variance of the residual turbulence which is respectively 1,7 and 21 time(s) the variance of the static aberrations.

Two simultaneous long-exposure images are simulated, with a phase diversity between these images consisting of a 1.814 radian defocus. The corresponding defocus distance is proportional to the square of the f-number of the system and, at $1.6 \mu \mathrm{m}$, is $2.9 \mathrm{~mm}$ for an $f / 15$ system such as $\mathrm{N}$ and $2 \mathrm{~cm}$ for an $f / 40$ system such as $S$. These images are simulated following two schemes: finite exposure time images are made of the summation of $N$ short exposures. The long-exposure OTF is then the sum of $N$ short-exposure OTFs, each of which being computed through Eq. (2), with a phase $\varphi$ composed of the sum of the static aberrations and of the instantaneous AO-corrected turbulent wavefront; the turbulent wavefront is randomly generated from a PSD that takes into account both the turbulence profile and the AO correction. In contrast, infinite exposure time images are not computed as an empirical average. Instead, the residual phase structure function $D_{\varphi}$ is computed from the PSD of the AO-corrected turbulent wavefront, then the ATF $\tilde{h}_{\mathrm{a}}$ is computed via Eq. (3), and the images $\boldsymbol{i}_{\mathrm{f}}$ and $\boldsymbol{i}_{\mathrm{d}}$ are computed according to Equation (6).

\subsection{Influence of exposure time}

We first consider the case of a high performance, $\mathrm{S}$-like correction. The bottom curve of Figure 1 shows the evolution of the reconstruction error with the number of exposures used for the simulation. The first points (from 10 to 1000 exposures) are simulated with a finite exposure time, whereas the last point (noted infinity on the $\mathrm{X}$-axis) is simulated with an infinite exposure time. Because the correlation time of corrected turbulence is typically 10 to 100 milliseconds depending on turbulence parameters 
and on the AO correction quality, the simulations with 1000 exposures correspond to an integration time between 10 and 100 seconds.

The reconstruction error decreases with the number of exposures, down to very weak values (less than 0.01 radian) for an infinite exposure time. As the number of exposures increases, Eq. (1) becomes more valid, turbulence residuals become better fitted by a modification of the estimated object only (into an object $\boldsymbol{o}^{\prime}=\boldsymbol{h}_{\mathrm{a}} \star \boldsymbol{o}$ ), and the estimated phase is eventually only the set of static aberrations. Incidentally, we have checked that for a single turbulent exposure, the phase estimated is the sum of the turbulent wavefront and the static aberrations.

This simulation study shows that the estimation of static aberrations from a single pair of turbulencedegraded images is possible. The quality of aberration reconstruction is directly linked to the convergence of the images towards long-time exposures. For the AO system and the level of static aberrations considered here, an integration time corresponding to a thousand independent turbulence realizations yields a phase estimation error of about 0.012 radian, close to that obtained with an infinite exposure time. At $1.6 \mu \mathrm{m}$, this number translates into a $3 \mathrm{~nm} \quad$ optical path difference. This precision is compatible with the $5-10 \mathrm{~nm}$ static aberration residual that is needed for the detection of warm Jupiters on an 8-meter telescope. Incidentally, we see that with less than 1000 exposures the required precision would not be obtained for such a mission.

\subsection{Influence of AO correction quality}

Figure 1 shows the evolution of the estimation error, for different correction qualities, obtained here simply by varying the number of actuators of the AO system, all other parameters being equal. One can see that as the correction quality degrades, the turbulence residuals are more important and thus the estimation of the static aberrations needs more exposures for the same error level. The estimation errors for an infinite exposure time are equivalent for all three correction qualities. A spectral analysis of the estimated aberrations yields more insight into the estimation quality and is given in [8].

In the case of a N correction with $14 \times 14$ actuators and 1000 exposures, the phase estimation error is about 0.13 radian, which at $2.2 \mu \mathrm{m}$ translates into a $45 \mathrm{~nm}$ optical path difference. This is almost three times smaller than the residual static aberrations of $120 \mathrm{~nm}$ measured on $\mathrm{N}$ $\mathrm{C} \quad$ after off-line phase diversity measurement and correction [2]. The on-line long-exposure phase diversity technique could thus be an attractive way to calibrate quasi-static aberrations on non-extreme AO systems too.

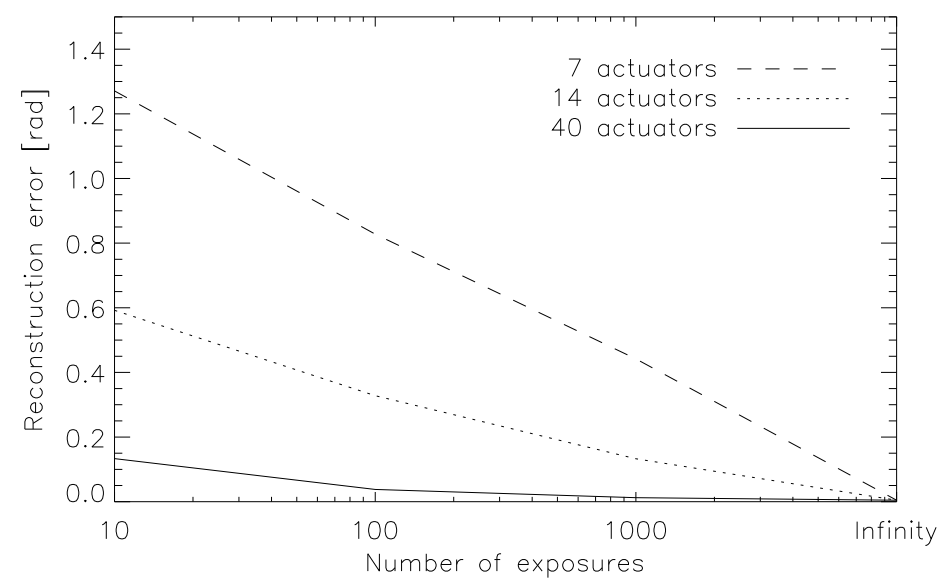

Fig. 1. Evolution of the reconstruction error with the exposure time (in number of independent turbulence realizations), for several levels of AO correction. Static aberrations are randomly generated according to conditions described in Subsection 4.1. 


\section{Acknowledgements}

The activities outlined in this communication have been partially funded as part of the European Commission, Seventh Framework Programme (FP7), Capacities Specific Programme, Research Infrastructures; specifically the FP7, Preparing for the Construction of the European Extremely Large Telescope Grant Agreement, Contract number INFRA-2007-2.2.1.28.

\section{Conclusion and perspectives}

We have validated by simulations an extension of the phase diversity technique that uses long exposure AO corrected images for sensing quasi-static aberrations.

The technical advantages of such a procedure are discussed in the text. From a system point of view, on-line long-exposure phase diversity opens a new area of applications, in particular it will allow one to correct in real time (meaning during the scientific exposure) for any evolution of instrumental defects. This may be considered for improvements to the $S$ instrument and should significantly improve the overall system detectivity. This technique can also be used as a phasing sensor for a segmented aperture telescope. Indeed, phase diversity can be applied to the peculiar aberrations constituted by the differential tip-tilts and pistons of such a telescope.

Thus, long-exposure phase diversity may be particularly useful for the future Planet Finder project on the E-ELT called EPICS. Indeed, on the one hand, for this project, the detectivity requirements are by far more stringent than for $\mathrm{S}$ and the on-line correction of non-common path aberrations is mandatory. And on the other hand, without any opto-mechanical modification of the sensor, longexposure phase diversity should enable very accurate measurements of the segments' phasing.

Short-term perspectives include a more thorough study of the performance of the long-exposure phase diversity technique, coupled with a global system analysis.

\section{References}

1. A. Blanc, T. Fusco, M. Hartung, L. M. Mugnier, and G. Rousset, "Calibration of NAOS and CONICA static aberrations. Application of the phase diversity technique," Astron. Astrophys. 399, 373-383 (2003).

2. M. Hartung, A. Blanc, T. Fusco, F. Lacombe, L. M. Mugnier, G. Rousset, and R. Lenzen, "Calibration of NAOS and CONICA static aberrations. Experimental results," Astron. Astrophys. 399, 385-394 (2003).

3. R. A. Gonsalves, "Phase retrieval and diversity in adaptive optics," Opt. Eng. 21(5), 829-832 (1982).

4. L. M. Mugnier, A. Blanc, and J. Idier, "Phase Diversity: a Technique for Wave-Front Sensing and for Diffraction-Limited Imaging," in Advances in Imaging and Electron Physics, P. Hawkes, ed., vol. 141, chap. 1, pp. 1-76 (Elsevier, 2006).

5. D. S. Acton, D. Soltau, and W. Schmidt, "Full-field wavefront measurements with phase diversity," Astron. Astrophys. 309, 661-672 (1996).

6. N. Baba and K. Mutoh, "Measurement of telescope aberrations through atmospheric turbulence by use of phase diversity," Appl. Opt. 40(4), 544-552 (2001).

7. D. J. Lee, B. M. Welsh, and M. C. Roggemann, "Diagnosing unknown aberrations in an adaptive optics system by use of phase diversity," Opt. Lett. 22(13), 952-954 (1997).

8. L. M. Mugnier, J.-F. Sauvage, T. Fusco, A. Cornia, and S. Dandy, "On-Line Long-Exposure Phase Diversity: a Powerful Tool for Sensing Quasi-Static Aberrations of Extreme Adaptive Optics Imaging Systems," Opt. Express 16(22), 18,406-18,416 (2008).

9. F. Roddier, ed., Adaptive Optics in Astronomy (Cambridge University Press, Cambridge, 1999).

10. R. G. Paxman, T. J. Schulz, and J. R. Fienup, "Joint estimation of object and aberrations by using phase diversity,” J. Opt. Soc. Am. A 9(7), 1072-1085 (1992).

11. A. Blanc, L. M. Mugnier, and J. Idier, "Marginal estimation of aberrations and image restoration by use of phase diversity," J. Opt. Soc. Am. A 20(6), 1035-1045 (2003).

12. J. Idier, L. Mugnier, and A. Blanc, "Statistical behavior of joint least square estimation in the phase diversity context,” IEEE Trans. Image Processing 14(12), 2107-2116 (2005).

13. L. Meynadier, V. Michau, M.-T. Velluet, J.-M. Conan, L. M. Mugnier, and G. Rousset, "Noise propagation in wave-front sensing with phase diversity," Appl. Opt. 38(23), 4967-4979 (1999).

14. L. Jolissaint, J.-P. Véran, and R. Conan, "Analytical modeling of adaptive optics: foundations of the phase spatial power spectrum approach,” J. Opt. Soc. Am. A 23(2), 382-394 (2006). 